\title{
PERI-LYMPH NODAL PATHOLOGICAL FIBROSIS AND AXILLARY SURGICAL IMPAIRMENT AFTER 14G PERCUTANEOUS FRAGMENT BIOPSY OF SENTINEL LYMPH NODE DETECTED WITH CONTRAST-ENHANCED ULTRASONOGRAPHY (CEUS) IN EARLY BREAST CANCER PATIENTS
}

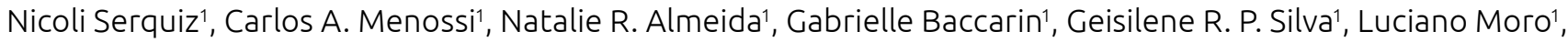
Sophie Derchain', Rodrigo M. Jales ${ }^{1}$

'Women's Hospital "Prof. Dr. José Aristodemo Pinotti", Universidade Estadual de Campinas - Campinas (SP), Brazil.

Objectives: To evaluate peri-lymph nodal pathological fibrosis in axillar surgical specimens of breast cancer patients submitted to $14 \mathrm{G}$ percutaneous fragment biopsy (PFB) of axillary sentinel lymph node identified with contrast-enhanced ultrasonography (CEUS). Methods: This study was originally designed to assess the performance of CEUS+PFB on the axillary staging of early-stage breast cancer. The selection of participants was interrupted, and the design was reformulated to this descriptive cross-sectional study after unexpected peri-lymph nodal pathological fibrosis reported in axillary surgery specimens. The frequency or means of the main clinical, ultrasonographic, surgical and pathological characteristics were calculated. T-test or Pearson Chi-Square test compared the groups of patients with and without peri-lymph nodal pathological fibrosis. Results: Forty-eight patients submitted to CEUS+PFB and axillary surgery were eligible for this study. Axillary surgical specimens showed peri-lymph nodal fibrosis in 9/48 (18.7\%) patients. The majority of peri-lymph nodal fibrosis were described as moderate $(4 / 9(44.4 \%))$ or severe $(4 / 9(44.4 \%))$. There was no significant difference between groups regarding patient age ( $p=0.99)$, breast tumor size $(p=0.60)$, rate of lymph-node metastasis $(p=0.83)$, use of aromatase inhibitor ( $p=0.43$ ), number of intradermal contrast injection ( $p=0.68)$, CEUS sentinel lymph identification ( $p=0.10)$, and CEUS sentinel lymph node mean maximum diameter $(\mathrm{p}=0.24)$. Axillary surgical impairment or hematoma were only reported in patients with axillary peri-lymph nodal fibrosis ( $\mathrm{p}<0.001$ and $\mathrm{p}=0.003$, respectively). Mean time between CEUS+PFB and axillary surgery was shorter $(\mathrm{p}=0.04)$ in patients with peri-lymph nodal fibrosis. Conclusion Peri-lymph nodal pathological fibrosis may impair the axillary surgical procedure of early breast cancer patients staged with CEUS+PFB. 